\title{
La evaluación de la «capacidad de la persona»: en la práctica actual y en el contexto del consentimiento informado
}

\author{
Gladys Bórquez Ea, Gina Raineri B ${ }^{b}$, Mireya Bravo $L^{a}$. \\ The evaluation of decision making \\ capacity in health care and its \\ relationship to informed consent
}

The decision making capacity of patients will acquire special relevance with the introduction of informed consent in clinical practice and the new normative that will appear in the future when the bills about health services are approved in the Congress. This paper reviews the concept of decision making capacity in the context of health care and its legal background in Chile. The main problems that arise from the comprehension, use and assessment of decision making capacity, are analyzed. The assessment of this capacity is, in most cases, subjective since there are no criteria, protocols or standards to be used. In courts of justice, the sexercise capacity» assessment is requested to experts. These specialized physicians will evaluate the mental health of the subject and its implications in the capacity to make decisions. In practice, it is difficult to integrate the concept of capacity from the law or health care perspective. It is concluded that the elaboration of criteria, standards and procedures to evaluate the decision capacity of patients, is mandatory (Rev Méd Chile 2004; 132: 1243-8).

(Key Words: Decision making; Ethics, medical; Informed consent)

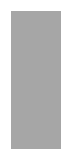

\begin{abstract}
Recibido el 10 de noviembre, 2003. Aceptado en versión corregida el 17 de agosto, 2004. Departamento de Bioética y Humanidades Médicas, Facultad de Medicina, Universidad de Chile. aMagíster Bioética

${ }^{\mathrm{b}}$ Abogado y Enfermera
\end{abstract}

\begin{abstract}
$\mathrm{C}$ on el nacimiento de la bioética, a partir de la década 1970-79, surge un nuevo estilo de hacer medicina, una relación diferente entre el enfermo y el profesional de la salud, entre cuyas características podemos destacar el convencimiento de que la manera más adecuada de abordar hoy este encuentro es a través de la participación y la deliberación conjunta.
\end{abstract}

Correspondencia a: Dra. Gladys Bórquez E. Fax: (56) (2) 7793850. E-mail: gborquez@chp.cl
El proceso de Consentimiento Informado traduce este nuevo estilo, constituyendo el núcleo de la moderna bioética, y se fundamenta en el reconocimiento del paciente como un sujeto moral racional con autonomía para gobernar su propia vida y muerte. Su esencia no es otra cosa que considerar que las decisiones en salud, para ser moralmente aceptables, deben ser decisiones autónomas tomadas por los afectados por ellas.

En salud, una decisión es autónoma cuando cumple tres condiciones: voluntariedad, informa- 
ción y capacidad ${ }^{1}$. De estos tres elementos tal vez el más difícil de valorar es la capacidad.

Con la introducción en la práctica clínica del Consentimiento Informado y en el futuro próximo de las normativas que emanen de los proyectos de leyes (actualmente en trámite) de \$erechos y deberes de las personas en salud»y de los Derechos sexuales y reproductivos de las personas», la correcta evaluación de la capacidad de los pacientes para tomar decisiones adquiere una importancia relevante.

Por lo anterior, nos pareció importante revisar tanto el concepto de capacidad sanitaria para la toma de decisiones, su evaluación y las propuestas para realizarla, como el de capacidad legal junto a las normativas legales y sanitarias vigentes en Chile. Analizamos además los problemas más importantes que se plantean en la práctica diaria que derivan de la comprensión, uso y evaluación de la capacidad.

\section{EL CONCEPTO DE CAPACIDAD PARA LA TOMA DE DECISIONES}

En el Diccionario de la Real Academia de la Lengua Española (2001) se define capacidad como saptitud, talento, cualidad que dispone a alguien para el buen ejercicio de algo».

En la literatura bioética, al evaluar la autonomía de los pacientes para tomar decisiones, se usa como sinónimos los términos capacidad y competencia, dependiendo de las fuentes bibliográficas consultadas.

En la literatura norteamericana se utiliza en forma habitual el término competency como aptitud para ejercer un derecho y capacity como aptitud para realizar determinada acción. Entre los autores españoles no existe acuerdo para el uso de estos términos, algunos, como Pablo Simon ${ }^{2}$, prefieren utilizar el término competencia para tomar decisiones en el campo sanitario, para diferenciarlo expresamente del término capacidad que utiliza el Derecho Español, donde se habla de «capacidad de obrar de hecho»y de «capacidad de obrar de derecho»la primera equivale a capacity y la segunda a competency; otros ${ }^{3}$ prefieren utilizar el término capacidad cuando se usa en el campo de la salud y existen aquéllos ${ }^{4}$ que utilizan capacidad y competencia como sinónimos.
En la literatura disponible en nuestro medio algunos autores utilizan indistintamente ambos términos, mientras otros hablan expresamente de capacidad $^{5-7}$.

El Derecho Civil Chileno, en tanto, distingue: capacidad de goce como la aptitud legal de las personas para adquirir derechos y obligaciones y capacidad de ejercicio como la aptitud de las personas para obrar por sí mismas sin el ministerio o autorización de otro, lo que supone la existencia de una voluntad capaz de discernir con responsabilidad y con libertad en actos jurídicos patrimoniales o extrapatrimoniales ${ }^{8}$.

Con el objeto de simplificar el problema de terminología en nuestro país, decidimos usar el término capacidad y hablar de capacidad sanitaria y de capacidad legal según el ámbito en el que se use.

\section{LA CAPACIDAD SANITARIA Y SU EVALUACIÓN}

El hecho que la capacidad sanitaria sea un elemento central en la práctica del proceso de Consentimiento Informado, hace que ésta sea para el profesional de la salud de una gran responsabilidad ética, fundamentada tanto en el principio de autonomía como en los principios de beneficencia y de no maleficencia 9 .

El respeto a la dignidad y libertad de las personas como agentes autónomos obliga a asegurar las condiciones necesarias para permitir que el paciente tome una decisión autónoma.

El fundamento ético de lo anterior, descansa, de acuerdo a Kant ${ }^{10}$, en que toda persona tiene un valor incondicional, es un fin en sí mismo y no sólo un medio» y a Stuart Mill ${ }^{11}$ en que ‘oda persona debe desarrollarse según sus convicciones personales siempre que no interfieran con la libertad del resto».

La capacidad para tomar decisiones en salud se define por la presencia de un cierto número de habilidades, fundamentalmente psicológicas (afectivas, cognitivas, volitivas) ${ }^{2}$ que permiten tomar en forma autónoma una decisión concreta en un momento determinado, no dice nada de la capacidad para tomar otras decisiones, ni es un juicio respecto de la capacidad legal. Así, se puede ser capaz para tomar decisiones de la vida diaria y no para decidir un tratamiento o participar en una investigación. 
Los criterios de capacidad sanitaria hacen referencia fundamentalmente a las aptitudes de los pacientes para recibir, comprender y procesar racionalmente información, tomar una decisión y comunicarla adecuadamente. Puede entenderse como un continuum entre dos puntos extremos: la capacidad plena y la incapacidad total, existiendo un punto de corte por debajo del cual se califica de incapaces a los sujetos, y por sobre el cual se les considera capaces. Los estándares exigidos no son siempre los mismos, sino que ellos se deben definir en función del tipo de decisión a tomar. Así el umbral de capacidad no es fijo sino móvil ${ }^{13}$.

Actualmente, a los pacientes se les reconoce autonomía para tomar decisiones si manifiestan capacidad para ello, actúan intencionalmente, en ausencia de influencias externas, y expresan su voluntad tomando en cuenta las consecuencias que dichas decisiones tienen para su salud y para su vida futura. Cumplidas las condiciones anteriores, el acto del médico será beneficente si respeta la autonomía del paciente. En cambio, el acto médico resultará maleficente cuando, sin una correcta evaluación de la capacidad, se permite a un paciente incapaz desde un punto de vista sanitario, que tome decisiones que pueden producirle daño. Frente a un paciente catalogado como incapaz para tomar decisiones es necesario entregar el poder de tomarla a un tercero (generalmente un familiar), ya sea en forma transitoria 0 definitiva, conforme lo señala la ley. Las decisiones así tomadas son las llamadas decisiones de sustitución o subrogadas, y deben responder al criterio del mejor interés del paciente ${ }^{14}$.

La evaluación de la capacidad sanitaria es, en la práctica diaria, en la mayoría de los casos subjetiva y por lo tanto prudencial, ya que aún no existen criterios (definición de habilidades y aptitudes a evaluar), estándares (grados de suficiencia a exigir) y protocolos (proceso para determinarla) consensuados para realizarla en forma objetiva y adecuada. Esta evaluación es, en la mayoría de los casos, de responsabilidad del médico tratante, y sólo cuando éste tiene dudas, consulta a un experto. En estos casos, el informe que el especialista entrega habitualmente corresponde a un diagnóstico de salud mental y no a un juicio respecto de la capacidad para participar o actuar en el proceso del Consentimiento Informado, por lo que no resulta vinculante para el médico tratante.

Desde la década del 70 en adelante han surgido diversas propuestas para evaluar la capacidad, casi todas provienen desde Estados Unidos $13,15-22$. En casi todas estas propuestas se definen criterios para evaluar (entre 3 y 9 criterios), pero sólo unas pocas definen estándares $^{13,17}$, los que en su mayoría están en relación a las consecuencias de las decisiones y sólo tres proponen un protocolo para determinarla ${ }^{20-22}$.

\section{EVALUACIÓN DE LA CAPACIDAD LEGAL}

El Código Civil de Chile señala que para que una persona se obligue a otra por un acto o declaración de voluntad, es necesario cumplir ciertos requisitos, y que toda persona es legalmente capaz, excepto aquellas que la ley declara incapaces y que cada incapaz de derecho o de hecho lo es únicamente en la medida establecida por la ley. Así, la incapacidad no puede extenderse por analogía. El mismo Código y su última modificación por la ley 19.904/2003, establece quiénes son incapaces de obrar, distinguiendo entre incapacidad de ejercicio absoluta y relativa.

Cuando los tribunales de justicia necesitan evaluar la capacidad de ejercicio de las personas recurren ordinariamente a los medios de prueba consagrados en la legislación. Uno de éstos, es el informe de peritos y se solicita cuando hay que evaluar la capacidad de la persona en diferentes ámbitos de su vida, es realizado por médicos especialistas y evalúa fundamentalmente el diagnóstico de salud mental en relación con una función determinada, de acuerdo a lo señalado por el magistrado. Este informe es uno de los antecedentes considerados por el juez al momento de dictar una sentencia judicial ${ }^{23}$.

\section{Normativa SANITARIA Y LEGAL EN CHILE}

El Código de Ética Médica del Colegio Médico de Chile A.G., de 1986, Art. 15 señala que: «..en caso de recurrir a tratamientos que entrañen riesgos ciertos o mutilación grave para el paciente, el 
médico deberá contar con el consentimiento expreso, dado con conocimiento de causa, por el enfermo o sus familiares responsables cuando sea menor de edad o esté incapacitado para decidir...» y el Art. 20: «.. señala que los informes médicos y peritajes médico-legales que las autoridades soliciten con el fin de evaluar grados de incapacidad, estado físico o mental, deberán ser evacuados por especialistas». Así se esboza cierta noción de que la capacidad está implícita en el consentimiento informado.

Lo anterior está refrendado en «El Decálogo de la Buena Práctica Médica del Colegio Médico de Chile A.G.»cuando dice en el punto 3: Entregar a mis pacientes información oportuna, clara y precisa, de manera que ellos puedan entender y decidir»y en el punto 4: «espetar el derecho de mis pacientes a participar de las decisiones que tengan relación con su salud».

Entre los decretos y resoluciones de la autoridad sanitaria destaca el Decreto 42/1986, del MINSAL que en su artículo 105 señala: «.. Los profesionales tratantes deberán informar, en lo posible y cuando proceda, a los pacientes, a sus representantes legales o a los familiares....», consagrando así el Consentimiento Informado junto con la posibilidad de rechazar tratamientos por parte del paciente o sus familiares, pero no se hace mención a la capacidad.

Para algunas situaciones clínicas particulares, como en los enfermos mentales existen referencias a la capacidad:

El Decreto 570/2000 de MINSAL, aprueba el Reglamento para la «nternación de las personas con enfermedades mentales y sobre los establecimientos que la proporcionan».

El decreto 633/2001 del MINSAL, crea una Comisión Nacional con el fin de supervisar las tareas relativas al respeto de los derechos de los pacientes hospitalizados en centros de internación psiquiátrica, a la aplicación de tratamientos psiquiátricos que requieran consentimientos o segunda opinión.

La Resolución Exenta 2326/2000 del MINSAL regula la esterilización voluntaria de personas en edad reproductiva.

La Resolución Exenta 656/2002 del MINSAL regula los tratamientos psicoquirúrgicos o de cirugía cerebral.
Para los discapacitados en general: Los Decretos 2505/1995 y 2542/1996 del MINSAL (Ley $18.600,19.735$ y 19.284) definen una normativa respecto de la discapacidad, se refieren sólo a aspectos formales de la evaluación, de la declaración y de la certificación de la discapacidad.

Para los adolescentes, se reconoce que gozan de la madurez suficiente para tomar decisiones en la esfera de los derechos subjetivos y personales como el ejercicio de su sexualidad. El Programa de Salud del Adolescente del MINSAL norma en relación con los temas de paternidad responsable y de contracepción.

Para los adultos mayores no existen reglamentaciones específicas ni las hay en la reciente creación por ley 19.828 del Servicio Nacional del Adulto Mayor.

Las normas citadas ${ }^{24-26}$ si bien consagran una reglamentación, no entregan propuestas para evaluar la capacidad (en lo que se refiere a criterios, estándares y protocolos).

Para los menores de edad la legislación general aplicable se encuentra en normas internacionales que nuestro país suscribe, como la Declaración Universal de Derechos Humanos, el Pacto Internacional de Derechos Civiles y Políticos de las Naciones Unidas, el Pacto de San José de Costa Rica ${ }^{27}$ y la Carta de Derechos del Niño ${ }^{28}$ las que consagran el derecho de los menores a las medidas de protección que su condición requiera, tanto por parte de su familia como de la sociedad y del estado, teniéndose debidamente en cuenta las opiniones del niño, en función de su edad y madurez.

El Código Civil de Chile plantea para el menor de edad la presunción general de incapacidad legal. En su artículo 26 distingue: niño al menor de 7 años; impúber, al varón menor de 14 años y a la mujer menor de 12 años; menor adulto, al que ha dejado de ser impúber; mayor de edad 0 simplemente mayor, al que ha cumplido 18 años; y menor de edad o simplemente menor, al que no ha llegado a cumplirlos.

También hay normas legales que reconocen la capacidad de ejercicio a menores de edad a partir de los 14 años en materias específicas, por ejemplo la que les permite efectuar giros desde cuentas de ahorro bancarias sin su representante legal.

Para los adolescentes hay organismos que les reconocen capacidad legal en determinadas mate- 
rias, como es el caso de Instituto Nacional de la Juventud que en el año 2002 publicó el «statuto Tipo de Corporaciones Juveniles»para las organizaciones de jóvenes desde los 15 años.

En materia penal la Ley de Menores 19.806/ 2002 menciona la evaluación de los menores de 18 años y mayores de 16 años, respecto de su capacidad de discernimiento, en materia penal.

En el caso de los menores adultos (mayores de 14), nos encontramos frente a una situación dicotómica, ya que si bien no son considerados plenamente capaces desde el punto de vista legal, sus actos pueden tener valor en ciertas circunstancias y bajo ciertos respectos, determinados por las leyes.

\section{ConCLUSIONES}

El estudio y revisión bibliográfica realizada en relación a la capacidad de los pacientes para tomar decisiones en materias de salud, nos han permitido constatar una preocupación creciente por su comprensión y evaluación, a la vez que la existencia de una serie de normativas legales y éticas que nacieron como consecuencia de dicha preocupación.

\section{REFERENCIAS}

1. Beauchamp T, Childress J. El Respeto a la Autonomía. En: Principios de Etica Biomédica. Barcelona: Masson S.A; 1999; 113-72.

2. Simón P. Capacidad o Competencia. En: El Consentimiento Informado. España: Triacastela; 2000; 337-8.

3. SÁncheZ M. Consentimiento Informado. En: Historia y teoría del Método de la Medicina. Introducción al Pensamiento Médico. Barcelona: Ediciones Masson; 1998; 436-7.

4. Gracia D. Bioética clínica. Etica y Vida. Estudios de Bioética 2. Colombia: El Buho; 1998.

5. Kotтow M. Etica Médica. En: Introducción a la Bioética. Chile: Editorial Universitaria; 1995; 1456.

6. Lolas F. Los principios fundamentales. En: Bioética. Chile: Editorial Universitaria; 1998; 51-2.
Dada la importancia de la incorporación del principio de autonomía de los pacientes en la práctica clínica, es necesario continuar con la reflexión de los diferentes aspectos que hay que considerar para su adecuada comprensión y respeto en la relación médico-paciente.

La capacidad de los pacientes para tomar decisiones es una condición necesaria, pero no suficiente, que permite a las personas ejercer su autonomía.

Con el objeto de realizar una adecuada evaluación y valoración de dicha capacidad, particularmente en el contexto del Consentimiento Informado, es necesario en nuestro medio, elaborar criterios, estándares y procedimientos de medición. Existen experiencias extranjeras en relación con test orientadores como el Minimental, que también aplican algunas personas en nuestro medio. Nosotros estamos iniciando un trabajo empírico, con cuyos resultados esperamos plantear criterios al respecto.

Trabajar en este sentido, sin duda aportará beneficios tanto a la sociedad, como para el establecimiento de futuras políticas de salud, y evitará los problemas que, derivados de una mala interpretación de los conceptos de autonomía, capacidad y derechos de los pacientes, crean frecuentes conflictos en la relación clínica.

7. Lavados M. La Noción de Competencia y su rol en las Decisiones Clínicas. Bol Escuela de Med PUC 1998; 27: 14-6.

8. Vial del Río V, Lyon A. Derecho Civil, Teoría General de los Actos Jurídicos y de Las Personas. Ediciones Universidad Católica de Chile 1985; 284-6.

9. Gracia D. Fundamentación y enseñanza de la Bioética. Etica y Vida. Estudios de Bioética 1. Colombia: El Buho; 1998.

10. Kant I. Fundamentación de la Metafísica de las Costumbres. Madrid: Espasa Calpe SA; 1995; 102-7.

11. MıL JS. Sobre la Libertad. Madrid: Orbis; 1985; 32.

12. Simon P. Capacidad o Competencia. En: El Consentimiento Informado España: Triacastela; 2000; 339.

13. Drane J. Competency to give an Informed Consent. A model for Making Clinical Assesments. JAMA 1984; 252: 925-7. 
14. Buchanan A, Brock D. Deciding for others. The ethics of surrogare decision making. New York: Oxford Univ Press; 1989.

15. Rотн L, Meisel A, Lidz C. Tests of competency to Consent to Treatment. Am J Psychiatry 1977; 134: 279-84.

16. Appelbaum P, Roth L Competency to Consent to Research. A Psychiatrics Overview. Arch Gen Psychiatry 1982; 39: 951-8.

17. President's Comission for the Study of Ethical Problems in Medicine and Biomedical and Behavioral Research. Making Health Care Decisions Vol 1 Washington DC: Goverment Printing Office, 1982.

18. WhITE BC, Competence to Consent. Washington DC: Georgetown Univ. Press, 1994; 154-84.

19. Alexander MP. Clinical Determination of Mental Competente. Arch Neurol 1988; 45: 23-6.

20. Kaplan K, Price M. The Clinican's Role in Competency Evaluations. Gen Hosp Psychiatry 1989; 11: 397-403.

21. Fitten L, Hamann C, Evans GL Relationship of Cognitive Impairment to Decision Making Competence in Nursing Home Residents: Develop- ment of a Competence Assesment Protocol. J Am Geriatr Soc 1984; 32 (Suppl): s19.

22. JanofsKY JS, McCartHY JS, Folstein MF. The Hopkins Competency Assesment Test: A Brief Method for Evaluating Patient's capacity to Give Informed Consent. Hosp. Community Psychiatry 1992; 43: 132-6.

23. Rомо O. Medicina Legal Elementos de Ciencias Forenses. Editorial Jurídica de Chile, 1992; 43-53.

24. Maturana A. Código Sanitario. Comentado, Concordado, Jurisprudencia, Leyes y Reglamentos Complementarios. Santiago de Chile: Editorial Jurídica Cono Sur Ltda; Tomo I 1998 y Tomo II 1999.

25. Biblioteca del Congreso Nacional de Chile. En http://www.congreso.cl (consultado el 5 de abril 2003).

26. Centro de Documentación del Ministerio de Salud de Chile En http://www.minsal.cl (consultado el 5 de abril, 2003)

27. Evans E. Los Derechos Constitucionales, Tomo I, Segunda Edición actualizada, Editorial Jurídica de Chile, 1999.

28. Derechos del Niño En http://boes.org/un/spaunb.html 